\title{
Trajectory Tracking Control of Parallel Manipulator Based on Udwadia-Kalaba Approach
}

\author{
Chenming Li, ${ }^{1}$ Han Zhao, ${ }^{1}$ Shengchao Zhen, ${ }^{1,2}$ Kang Huang, ${ }^{1}$ Hao Sun, ${ }^{1,2}$ \\ Ke Shao, ${ }^{1}$ and Bin Deng ${ }^{1}$ \\ ${ }^{1}$ School of Mechanical Engineering, HeFei University of Technology, Hefei 230009, China \\ ${ }^{2}$ School of Mechanical Engineering, Georgia Institute of Technology, Atlanta 30332, USA \\ Correspondence should be addressed to Shengchao Zhen; zhenshengchao@qq.com
}

Received 1 August 2017; Accepted 27 November 2017; Published 14 December 2017

Academic Editor: Emiliano Mucchi

Copyright (C) 2017 Chenming Li et al. This is an open access article distributed under the Creative Commons Attribution License, which permits unrestricted use, distribution, and reproduction in any medium, provided the original work is properly cited.

\begin{abstract}
There have been many approaches for achieving the trajectory tracking control of parallel manipulator. However, these approaches are complex for calculating Lagrangian multipliers. In this paper, unlike the former approaches, a new approach of trajectory tracking control which is based on Udwadia-Kalaba approach is presented. Using this methodology, we can obtain a concise and explicit equation of motion and consider holonomic and nonholonomic constraint whether it is ideal or nonideal simultaneously. The most important difference is that we divide constraints into structural constraints and performance constraints in this paper. Structural constraints are used to establish dynamic model without regard for trajectory control. And performance constraints are used to represent the desired trajectory. For the parallel manipulator, a nonlinear dynamics system, its constraint forces are obtained by second-order constraints. And the numerical simulation in MATLAB shows the parallel manipulator's movement meets the requirement; tracking trajectory is exact and perfect. Through this paper, we can see that the method can simplify calculation availably.
\end{abstract}

\section{Introduction}

Parallel manipulator is a kind of closed-loop kinematic chain mechanism in which the end-effector is linked to the base by several independent kinematic chains [1]. In comparison with the serial manipulator which has openloop structure, the parallel manipulator has many advantages, such as higher stiffness, higher accuracy, higher payload capacity, lower inertia, and smaller workspace. Therefore, it has attracted a great amount of attention and interest among the researchers and engineers and has been applied to many industrial applications in the past decade, like milling [2], rapid machining [3], medical resuscitation [4], and so on.

In order to achieve the great potential performances existing in the parallel manipulator system, the controller design is essential and indispensable. To deal with the trajectory tracking control problem of the parallel manipulator, it is easy to find that many advanced controllers have been proposed and successfully implemented, like proportional-integralderivative control [5], sliding mode control [6], adaptive control $[7,8]$, robust control $[9,10]$, robust PID control [11], adaptive sliding mode control [12], adaptive robust control [13], fuzzy control [14] neural network control [15], fuzzy neural network control [16], and so on.

Parallel manipulators can be designed with various mechanical structures because the designers want them to realize different control requirements according to the actual demands. In this paper, a 3-degree-of-freedom (3-DOF) planar parallel manipulator (3-HSS, H-helix pair, S-spherical pair) has been designed and constructed. To deal with the trajectory tracking control problem of this manipulator, our methodology is based on a novel, concise approach to explicitly formulate the equations of motion for constrained systems proposed by Udwadia and Kalaba [17-20].

In this paper, we will use the basic kinematics equations proposed by Udwadia and Kalaba based on Gauss's theory for modeling and trajectory tracking of the parallel manipulators. There are several key points by using the method in this paper compared with the traditional control method. First, there is no need to obtain the complex Lagrangian multipliers during the process of dynamic modeling of the parallel manipulators. Second, we define the constraint equations by a new perspective which means the constraints are divided into structural constraints and performance constraints. 
Structural constraints are used to set up the dynamic model in the absence of trajectory control. And performance constraints are used to reflect the desired trajectory of the parallel manipulator. Third, we can obtain the needed motor torque for realizing the trajectory of manipulator directly by calculating the Udwadia and Kalaba equation. At last, the simulation results in this paper show that this method can control the parallel manipulator accurately.

\section{Udwadia and Kalaba Equation for Constrained Mechanical Systems}

In this section, we talk about the fundamental equation, called Udwadia-Kalaba equation, for constrained mechanical system. We can obtain the explicit closed-form equation of motion of the constrained mechanical system in three steps [18].

First, we consider the unconstrained mechanical system in which the coordinates are all assumed to be independent of each other. The equation of motion of this system can be expressed, by using Newtons or Lagranges equation, as

$$
M(q, t) \ddot{q}=Q(q, \dot{q}, t)
$$

with the initial conditions

$$
\begin{aligned}
& q(t=0)=q_{0}, \\
& \dot{q}(t=0)=\dot{q}_{0}
\end{aligned}
$$

where $q$ is the generalized coordinate $n$-vector and $t$ is the time; $M(q, t)$ is a positive definite inertia $n \times n$ matrix; $\dot{q}$ is an $n$-vector of velocity; $\ddot{q}$ is an $n$-vector of acceleration; $Q(q, \dot{q}, t)$ is an $n$-vector called the given force which could include centrifugal force, gravitational force, and control input. From (2), the generalized acceleration of the unconstrained system is given by

$$
\ddot{q}=a(q, \dot{q}, t)=M^{-1}(q, t) Q(q, \dot{q}, t) .
$$

Second, a set of control requirement or trajectory requirements is imposed as constraints on this uncontrolled system. We shall assume that the system is subjected to $h$ holonomic constraints of the form

$$
\varphi_{i}(q, t)=0, \quad i=1,2, \ldots, h ;
$$

meanwhile there are $m-h$ nonholonomic constraints in the form of

$$
\dot{\varphi}_{i}(q, \dot{q}, t)=0, \quad i=h+1, h+2, \ldots, m .
$$

Then, we can differentiate these constraint equations in Lagrangian mechanics which are usually in Pfaffian form. Under the assumption of sufficient smoothness, 2-order constraint equations can be acquired by taking the time derivative once on nonholonomic constraints and twice on holonomic constraints, which can be written in the form of matrix as

$$
A(q, \dot{q}, t) \ddot{q}=b(q, \dot{q}, t)
$$

where $A(q, \dot{q}, t)$ is an $m \times n$ matrix denoting the constraint matrix and $b(q, \dot{q}, t)$ is an $m \times 1$ vector.
Remark 1. The constraint is in either the zeroth-order or firstorder form which is expressed in the form of Lagrangian equation, Maggi equation, Boltzmann and Hamel equation, and Gibbs and Appell equation. However, for further dynamic analysis and control design, second-order constraints are considered to be the most suitable form. The reason is that the acceleration will be linear by using the secondorder constraint. And there is no need to worry about the loss of information during the differentiation (e.g., a constant). In fact, making the zeroth-order or holonomic constraint meet the initial condition means the initial condition retained the missing information. Thus, when we use Udwadia-Kalaba equation, the first step is to find all constraints including holonomic constraints and nonholonomic constraints in the unconstrained system. Then, we could obtain the secondorder form of constraints by differentiating the constraints with respect to time once or twice. Finally, we could transform these constraints into second-order matrix equations like (6).

Eventually, the explicit equations of motion with constraints will be formed. Notice that the additional generalized forces of constraints need to be imposed on the system as a result of the presence of constraints. We therefore assume the actual explicit equation of motion of the constrained system in the form of

$$
M(q, t) \ddot{q}=Q(q, \dot{q}, t)+Q^{C}(q, \dot{q}, t)
$$

where $Q^{C}(q, \dot{q}, t) \in R^{n}$ is the additional generalized force which is imposed on the unconstrained system, arising by the holonomic and nonholonomic constraints denoting a $n$ by 1 constraint matrix. Determining the general explicit form of $Q^{C}(q, \dot{q}, t) \in R^{n}$ is our goal with the $Q(q, \dot{q}, t)$ known at any time $t$. In Lagrangian mechanics, $Q^{C}(q, \dot{q}, t) \in R^{n}$ is considered to be ideal constraints because this kind of forces of constraint does zero work under virtual displacement according to the D' Alembert's Principle. However nonideal constraints also exist in an actual mechanical system, and the nonideal constraint forces including friction force, electromagnetic force, will be generated. Udwadia expressed $Q^{C}(q, \dot{q}, t) \in R^{n}$ in the form of

$$
Q^{C}(q, \dot{q}, t)=Q_{\mathrm{id}}^{C}(q, \dot{q}, t)+Q_{\mathrm{nid}}^{C}(q, \dot{q}, t)
$$

where $Q_{\mathrm{id}}^{C}(q, \dot{q}, t)$ represents the ideal constraint force and $Q_{\text {nid }}^{C}(q, \dot{q}, t)$ represents the nonideal constraint.

Furthermore, Udwadia and Kalaba have proved the general "explicit" equations of ideal and nonideal constraint forces at any time $t$ can be expressed in the form of

$$
\begin{aligned}
Q_{\mathrm{id}}^{C}(q, \dot{q}, t) & =M^{1 / 2} B^{+}\left(b-A M^{-1} Q\right) \\
Q_{\text {nid }}^{C}(q, \dot{q}, t) & =M^{1 / 2}\left(I-B^{+} B\right) M^{-1 / 2} c
\end{aligned}
$$

where $B=A M^{-1 / 2}$ and the superscript "+" denotes the Moore-Penrose generalized inverse. 
From (7), (8), and (9), the general "explicit" equation of motion can be expressed as

$$
\begin{aligned}
M \ddot{q}= & Q+M^{1 / 2} B^{+}\left(b-A M^{-1} Q\right) \\
& +M^{1 / 2}\left(I-B^{+} B\right) M^{-1 / 2} c .
\end{aligned}
$$

Remark 2. In particular, $c(q, \dot{q}, t)$ is a suitable $n$-vector in (10) which is determined by the mechanical system. Now we define a given nonzero $n$-vector virtual displacement $v$ in a constrained system at the instant time $t$ and the work done $W$ generated by $c(q, \dot{q}, t)$ under virtual displacement $v$. And the work done $W=v^{T} c(q, \dot{q}, t)$ which in any displacement $v$ which is subject to $A(q, \dot{q}, t) v=0$ is the same as the work done by $Q^{C}(q, \dot{q}, t) \in R^{n}$. Hence, we put the equation in form of

$$
W=v^{T} Q(q, \dot{q}, t)=v^{T} c(q, \dot{q}, t)
$$

which means we extend Lagrange's form of D' Alembert's Principle. The work done by the ideal constraint force $Q_{\mathrm{id}}^{C}(q, \dot{q}, t)$ and the nonideal constraint force $Q_{\text {nid }}^{C}(q, \dot{q}, t)$ under virtual displacements is

$$
\begin{aligned}
& v^{T} Q_{\text {id }}^{C}=0 \\
& v^{T} Q_{\text {nid }}^{C} \neq 0
\end{aligned}
$$

when the constraints are ideal and the total work done under virtual displacement is zero which means $c(q, \dot{q}, t)$ equals zero; according to D' Alembert's Principle, (10) becomes

$$
M \ddot{q}=Q+M^{1 / 2} B^{+}\left(b-A M^{-1} Q\right) .
$$

Therefore, at any instant of time, the constrained system is subjected to an additional constraint force $F^{C}(t)$, given by

$$
F^{C}(t)=M^{1 / 2} B^{+}\left(b-A M^{-1} Q\right) .
$$

If the unconstrained acceleration $M^{-1} Q$ is zero with the constant diagonal matrix $M=m I$ (14) becomes

$$
F^{C}(t)=m A^{+} b .
$$

Remark 3. There are two main advantages of this equation which Udwadia and Kalaba have provided. First, by using this equation, there is no need to determine the Lagrangian multipliers which are always complex. On the contrary, the parameters in the equation are all simple to obtain. Second, this equation applies to all holonomic and/or nonholonomic constrained systems including ideal or nonideal constraints.

\section{Trajectory Tracking Control of the Parallel Manipulator}

3.1. Dynamic Model of the Parallel Manipulator. The 3-HSS parallel manipulator is composed of a moving platform and three sets of sliding saddle chain structure. Each branched chain includes two equilong and parallel bars. One end of the

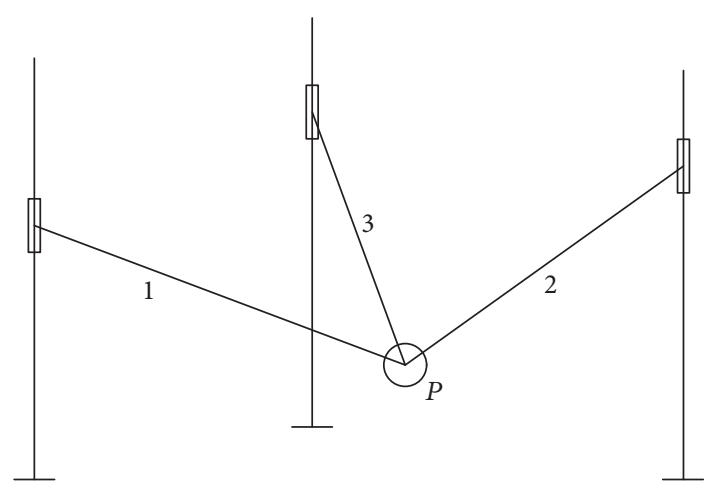

FIGURE 1: The 3-HSS mechanism structure.

bar is connected with the saddle by rolling spherical joint, and the other end is connected to the moving platform. As for the sliding saddle which is driven by the servomotor-screw-nut pair, it is moving along the ball screw which is mounted on the guide block. Hence the moving platform could achieve the 3D-motion. In addition, the platform is capable of high speed machining with the high speed motorized spindle on it.

The law of movement of each bar in branched chain is the same based on the parallelogram strut structure. Therefore, in this paper, we abstract the original mechanism into singlebar equivalent model as shown in Figure 1 during the kinematics modeling process. In this equivalent mechanism, each branched chain contains only one bar. And two ends of the bar are connected with the moving platform and the saddle by Hooke joint. Beside the saddle is driven by active pair (helix pair). Based on the above structure, the equivalent mechanism has the same degree of freedom as the original mechanism.

Next, we employ the classic universal joint, prismatic joint and spherical joint to illustrate the dynamic modeling of the delta parallel structure (as show in Figure 1). Following the procedure, we segment the delta parallel structure into four subsystems: three arm subsystems $(1,2,3)$ and one platform subsystem $(P)$. We shall then derive the equations of motion of the "unconstrained" subsystems.

Select reference frame $o x y z$. And assume the mechanism fixed on the plane oxy. Then consider the arm-subsystem in Figure 1 separately. Denote the following.

As shown in Figure 2, the end of the branched chain which is connected with the moving platform can be described with coordinates $q_{i}=\left[h_{i}, \theta_{i}, \varphi_{i}\right]^{T}, i=1,2,3$. And the moving platform can be described with coordinates $q_{P}=\left[x_{P}, y_{P}, z_{P}\right]^{T}$.

$h_{i}$ is height of the other end of the $i$ th branched chain. $\theta_{i}$ is the angle between $i$ th branched chain and $z$-axis. $\varphi_{i}$ is the angle between the $i$ th branched chain's projection in the oxy plane and $x$-axis. $l_{i}$ is the length of the $i$ th branched chain.

Thus, when we consider the arm-subsystem separately, the motor's coordinate of location can be expressed as $(0,0, h)$ and the end of the branched chain's coordinate of location can be expressed as $((l / 2) \sin \theta \cos \varphi,(l / 2) \sin \theta \sin \varphi$, 


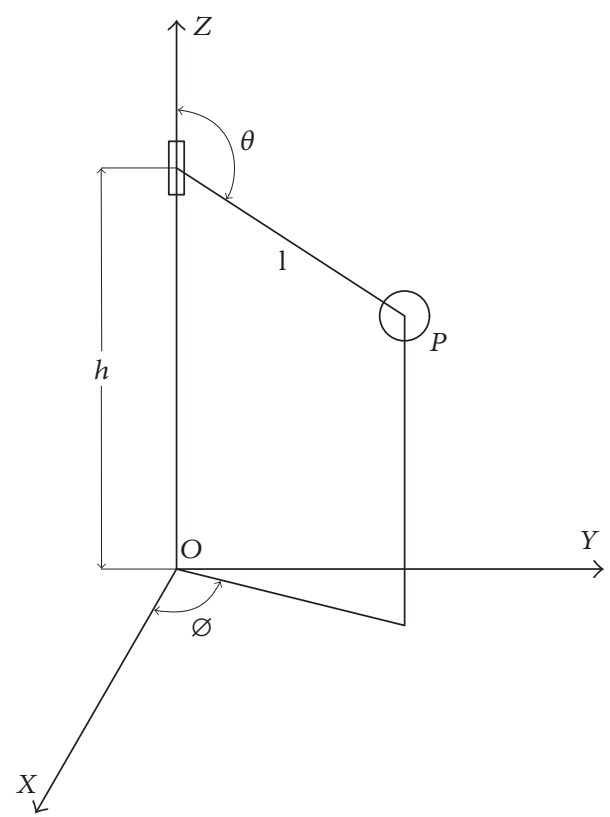

FIGURE 2: The arm-subsystem.

$h+(l / 2) \cos \theta)$. Then the kinetic and potential energy of the system can be written as

$$
\begin{aligned}
T_{s} & =T_{1}+T_{2}=\frac{1}{2} m_{1} v_{1}^{2}+\frac{1}{2} m_{2} v_{2}^{2}+\frac{1}{2} J \omega^{2}=\frac{1}{2} m_{1} \dot{h}^{2} \\
& +\frac{1}{2} m_{2}\left(\left(\frac{l}{2}(\dot{\theta} \cos \theta \cos \varphi-\dot{\varphi} \sin \theta \sin \varphi)\right)^{2}\right. \\
& +\left(\frac{l}{2}(\dot{\theta} \cos \theta \sin \varphi+\dot{\varphi} \sin \theta \cos \varphi)\right)^{2} \\
& \left.+\left(\dot{h}-\frac{l}{2} \dot{\theta} \sin \theta\right)^{2}\right)+\frac{1}{2} J\left(\dot{\theta}^{2}+\dot{\varphi}^{2}\right) \\
V_{s} & =V_{1}+V_{2}=m_{1} g h+m_{2} g\left(h+\frac{l}{2} \cos \theta\right)
\end{aligned}
$$

where $T_{s}, V_{s}$ is the system's kinetic/potential energy, $T_{1}, V_{1}$ is the motor's kinetic/potential energy, $T_{2}, V_{2}$ is the branched chain's kinetic/potential energy, and $m_{1}, m_{2}$ is the mass of motor/branched chain.

Next, adopting the standard Lagrangian approach, we have the following equation of motion for each "unconstrained" arm subsystem $(i=1,2,3)$ :

$$
M_{i}\left(q_{i}, t\right) \cdot \ddot{q}+C_{i}\left(q_{i}, \dot{q}_{i}, t\right)=\tau_{i}(t)
$$

where

$$
M_{i}=\left[\begin{array}{ccc}
m_{1}+m_{2} & -\frac{1}{2} m_{2} l \sin \theta_{1} & 0 \\
-\frac{1}{2} m_{2} l \sin \theta_{1} & J_{1}+\frac{1}{4} m_{2} l^{2} & 0 \\
0 & 0 & J_{1}+\frac{1}{4} m_{2} l^{2}
\end{array}\right]
$$

$$
\begin{aligned}
C_{i} & =\left[\begin{array}{c}
-\frac{1}{2} m_{2} l \dot{\theta}_{1}^{2} \cos \theta_{1}-\left(m_{1}+m_{2}\right) g \\
-m_{2} \dot{h}_{1} \dot{\theta}_{1} l \cdot \cos \theta_{1}+\frac{1}{2} m_{2} g l \sin \theta_{1} \\
0
\end{array}\right], \\
\tau_{i} & =\left[\begin{array}{c}
f_{a c i} \\
0 \\
0
\end{array}\right] .
\end{aligned}
$$

For the "unstrained" platform, that is, the subsystem 4 simplified as mass, by choosing the system coordinates as in Figure 2, the equation of motion is

$$
M_{i}\left(q_{i}, t\right) \cdot \ddot{q}+C_{i}\left(q_{i}, \dot{q}_{i}, t\right)=\tau_{i}(t), \quad i=p .
$$

We have

$$
\left[\begin{array}{ccc}
m_{p} & 0 & 0 \\
0 & m_{p} & 0 \\
0 & 0 & m_{p}
\end{array}\right]\left[\begin{array}{c}
\ddot{x}_{p} \\
\ddot{y}_{p} \\
\ddot{z}_{p}
\end{array}\right]=\left[\begin{array}{c}
0 \\
0 \\
m_{p} g
\end{array}\right] .
$$

So, the dynamic equations of the system is

$$
M(q) \ddot{q}+C(q, \dot{q})+G(q)=\tau .
$$

Now we cluster all the 4 subsystems together by letting

$$
\begin{aligned}
M & =\left[\begin{array}{llll}
M_{1} & & & \\
& M_{2} & & \\
& & M_{3} & \\
& & & M_{P}
\end{array}\right]_{12 \times 12}, \\
C & =\left[\begin{array}{l}
C_{1} \\
C_{2} \\
C_{3} \\
C_{P}
\end{array}\right]_{12 \times 1}, \\
\tau & =\left[\begin{array}{l}
\tau_{1} \\
\tau_{2} \\
\tau_{3} \\
\tau_{P}
\end{array}\right]_{12 \times 1},
\end{aligned}
$$

where

$$
M_{1}=\left[\begin{array}{ccc}
m_{1}+m_{2} & -\frac{1}{2} m_{2} l \sin \theta_{1} & 0 \\
-\frac{1}{2} m_{2} l \sin \theta_{1} & J_{1}+\frac{1}{4} m_{2} l^{2} & 0 \\
0 & 0 & J_{1}+\frac{1}{4} m_{2} l^{2}
\end{array}\right],
$$




$$
\begin{aligned}
& M_{2}=\left[\begin{array}{ccc}
m_{1}+m_{2} & -\frac{1}{2} m_{2} l \sin \theta_{1} & 0 \\
-\frac{1}{2} m_{2} l \sin \theta_{1} & J_{1}+\frac{1}{4} m_{2} l^{2} & 0 \\
0 & 0 & J_{1}+\frac{1}{4} m_{2} l^{2}
\end{array}\right] \text {, } \\
& M_{3}=\left[\begin{array}{ccc}
m_{1}+m_{2} & -\frac{1}{2} m_{2} l \sin \theta_{1} & 0 \\
-\frac{1}{2} m_{2} l \sin \theta_{1} & J_{1}+\frac{1}{4} m_{2} l^{2} & 0 \\
0 & 0 & J_{1}+\frac{1}{4} m_{2} l^{2}
\end{array}\right] \text {, } \\
& M_{P}=\left[\begin{array}{lll}
m_{p} & & \\
& m_{p} & \\
& & m_{p}
\end{array}\right] \text {, } \\
& C_{1}=\left[\begin{array}{c}
-\frac{1}{2} m_{2} l \dot{\theta}_{1}^{2} \cos \theta_{1}-\left(m_{1}+m_{2}\right) g \\
-m_{2} \dot{h}_{1} \dot{\theta}_{1} l \cdot \cos \theta_{1}+\frac{1}{2} m_{2} g l \sin \theta_{1} \\
0
\end{array}\right] \text {, } \\
& C_{2}=\left[\begin{array}{c}
-\frac{1}{2} m_{2} l \dot{\theta}_{1}^{2} \cos \theta_{1}-\left(m_{1}+m_{2}\right) g \\
-m_{2} \dot{h}_{1} \dot{\theta}_{1} l \cdot \cos \theta_{1}+\frac{1}{2} m_{2} g l \sin \theta_{1} \\
0
\end{array}\right], \\
& C_{3}=\left[\begin{array}{c}
-\frac{1}{2} m_{2} l \dot{\theta}_{1}^{2} \cos \theta_{1}-\left(m_{1}+m_{2}\right) g \\
-m_{2} \dot{h}_{1} \dot{\theta}_{1} l \cdot \cos \theta_{1}+\frac{1}{2} m_{2} g l \sin \theta_{1} \\
0
\end{array}\right], \\
& C_{P}=\left[\begin{array}{c}
0 \\
0 \\
-m_{p} g
\end{array}\right] \text {. }
\end{aligned}
$$

3.2. Structural Constraint of the Parallel Manipulator. We now introduce all the kinematic constraints among the subsystems and convert them into the second-order form.

Since the spherical joint only fixes the three translational DOF's between the connected links, we have the kinematic constraints, for $i=1,2,3, P$ as follows:

$$
\begin{aligned}
& x_{h 1}=x_{p}, \\
& x_{h 2}=x_{p}, \\
& x_{h 3}=x_{p} \\
& y_{h 1}=y_{p}, \\
& y_{h 2}=y_{p}, \\
& y_{h 3}=y_{p} \\
& z_{h 1}=z_{p},
\end{aligned}
$$

$$
\begin{aligned}
& z_{h 2}=z_{p}, \\
& z_{h 3}=z_{p} .
\end{aligned}
$$

Choosing generalized coordinate system

$$
q=\left[h_{1}, \theta_{1}, \varphi_{1}, h_{2}, \theta_{2}, \varphi_{2}, h_{3}, \theta_{3}, \varphi_{3}, x_{p}, y_{p}, z_{p}\right]^{T}
$$

So, we can get

$$
\begin{aligned}
& x_{p}=l \sin \theta_{1} \cos \varphi_{1}=l \sin \theta_{2} \cos \varphi_{2}=l \sin \theta_{3} \cos \varphi_{3} \\
& y_{p}=l \sin \theta_{1} \sin \varphi_{1}=l \sin \theta_{2} \cos \varphi_{2}=l \sin \theta_{3} \cos \varphi_{3} \\
& z_{p}=h+l \cos \theta_{1}=h+l \cos \theta_{2}=h+l \cos \theta_{3} .
\end{aligned}
$$

Differentiating (26) with respect to $t$ twice, we can get

$$
\begin{aligned}
\frac{1}{l} \ddot{x}_{p}= & \ddot{\theta}_{i} \cos \theta_{i} \cos \varphi_{i}-\ddot{\varphi}_{i} \sin \theta_{i} \sin \varphi_{i} \\
& -\dot{\theta}_{i}^{2} \sin \theta_{i} \cos \varphi_{i}-\dot{\varphi}_{i}^{2} \sin \theta_{i} \cos \varphi_{i} \\
& -2 \dot{\theta}_{i} \dot{\varphi}_{i} \cos \theta_{i} \sin \varphi_{i} \\
\frac{1}{l} y_{p}= & \ddot{\theta}_{i} \cos \theta_{i} \sin \varphi_{i}+\ddot{\varphi}_{i} \sin \theta_{i} \cos \varphi_{i} \\
& -\dot{\theta}_{i}^{2} \sin \theta_{i} \sin \varphi_{i}-\dot{\varphi}_{i}^{2} \sin \theta_{i} \sin \varphi_{i} \\
& +2 \dot{\theta}_{i} \dot{\varphi}_{i} \cos \theta_{i} \cos \varphi_{i} \\
\ddot{z}_{p}= & \ddot{h}-\ddot{\theta}_{i} l \sin \theta_{i}-\dot{\theta}_{i}^{2} l \cos \theta_{i}
\end{aligned}
$$

$$
i=1,2,3 \text {. }
$$

Let $i=1,(27)$ be written as matrix in the form of $A \ddot{q}=b$ :

$$
\begin{aligned}
& A_{1}=\left[\begin{array}{cccccc}
\frac{1}{l} & 0 & 0 & 0 & -\cos \theta_{1} \cos \varphi_{1} & \sin \theta_{1} \sin \varphi_{1} \\
0 & \frac{1}{l} & 0 & 0 & -\cos \theta_{1} \sin \varphi_{1} & -\sin \theta_{1} \cos \varphi_{1} \\
0 & 0 & 1 & -1 & l \sin \theta_{1} & 0
\end{array}\right], \\
& \ddot{q}_{1}=\left[\ddot{x}_{p}, \ddot{y}_{p}, \ddot{z}_{p}, \ddot{h}_{1}, \ddot{\theta}_{1}, \ddot{\varphi}_{1}\right]^{T},
\end{aligned}
$$

$b_{1}$

$$
=\left[\begin{array}{c}
-\dot{\theta}_{1}^{2} \sin \theta_{1} \cos \varphi_{1}-\dot{\varphi}_{1}^{2} \sin \theta_{1} \cos \varphi_{1}-2 \dot{\theta}_{1} \dot{\varphi}_{1} \cos \theta_{1} \sin \varphi_{1} \\
-\dot{\theta}_{1}^{2} \sin \theta_{1} \sin \varphi_{1}-\dot{\varphi}_{1}^{2} \sin \theta_{1} \sin \varphi_{1}+2 \dot{\theta}_{1} \dot{\varphi}_{1} \cos \theta_{1} \cos \varphi_{1} \\
-\dot{\theta}_{1}^{2} l \cos \theta_{1}
\end{array}\right] .
$$

Assume matrix, $i=1,2,3$ :

$A_{i}=\left[\begin{array}{ccc}0 & -\cos \theta_{i} \cos \varphi_{i} & \sin \theta_{i} \sin \varphi_{i} \\ 0 & -\cos \theta_{i} \sin \varphi_{i} & -\sin \theta_{i} \cos \varphi_{i} \\ -1 & l \sin \theta_{i} & 0\end{array}\right]$ 


$$
\begin{aligned}
& l=\left[\begin{array}{ccc}
\frac{1}{l} & 0 & 0 \\
0 & \frac{1}{l} & 0 \\
0 & 0 & 1
\end{array}\right] \\
& b_{i} \\
& =\left[\begin{array}{c}
-\dot{\theta}_{i}^{2} \sin \theta_{i} \cos \varphi_{i}-\dot{\varphi}_{i}^{2} \sin \theta_{i} \cos \varphi_{i}-2 \dot{\theta}_{i} \dot{\varphi}_{i} \cos \theta_{i} \sin \varphi_{i} \\
-\dot{\theta}_{i}^{2} \sin \theta_{i} \sin \varphi_{i}-\dot{\varphi}_{i}^{2} \sin \theta_{i} \sin \varphi_{i}+2 \dot{\theta}_{i} \dot{\varphi}_{i} \cos \theta_{i} \cos \varphi_{i} \\
-\dot{\theta}_{i}^{2} l \cos \theta_{i}
\end{array}\right] .
\end{aligned}
$$

The total parallel manipulator system can be written in a matrix form:

$$
\begin{aligned}
A_{S} & =\left[\begin{array}{llll}
l & A_{1} & & \\
l & & A_{2} & \\
l & & A_{3}
\end{array}\right]_{9 \times 12} \\
\ddot{q} & =\left[\ddot{x}_{p}, \ddot{y}_{p}, \ddot{z}_{p}, \ddot{h}_{1}, \ddot{\theta}_{1}, \ddot{\varphi}_{1}, \ddot{h}_{2}, \ddot{\theta}_{2}, \ddot{\varphi}_{2}, \ddot{h}_{3}, \ddot{\theta}_{3}, \ddot{\varphi}_{3}\right]^{T}, \\
b_{S} & =\left[\begin{array}{c}
b_{1} \\
b_{2} \\
b_{3}
\end{array}\right]_{9 \times 1} .
\end{aligned}
$$

\section{Trajectory Tracking Simulation of the Parallel Manipulator}

In this section, we aim to use the Udwadia-Kalaba approach to obtain an explicit, closed-form expression for the control force required to precisely meet the trajectory requirements of the 3 -HSS parallel manipulator.

\subsection{Performance Constraint of the Parallel Manipulator}

Case 1. Assume that the prespecified trajectory of the mobile platform is represented by the following equation:

$$
\begin{aligned}
& x_{p}=-\frac{2 \sqrt{3}}{3}-R \cos (w t) \\
& y_{p}=2+R \sin (w t) \\
& z_{p}=0.05 t .
\end{aligned}
$$

Differentiating the constraint equation (31) with respect to $t$ twice, we can get

$$
\begin{aligned}
& \ddot{x}_{p}=-R w^{2} \cos (w t) \\
& \ddot{y}_{p}=-R w^{2} \sin (w t) \\
& \ddot{z}_{p}=0 .
\end{aligned}
$$
of

Hence the system constraints can be written in the form

$$
A_{p}(q, t) \ddot{q}=b_{p}(q, \dot{q}, t)
$$

where

$$
\begin{aligned}
A_{P} & =\left[\begin{array}{lll}
1 & 0 & 0 \\
0 & 1 & 0 \\
0 & 0 & 1
\end{array}\right], \\
b_{P} & =\left[\begin{array}{c}
-R w^{2} \cos (w t) \\
-R w^{2} \sin (w t) \\
0
\end{array}\right] .
\end{aligned}
$$

Equation (31) is the performance constraints in this paper. Thus, the three servomotors which are on the saddle must make the platform satisfy (31) by controlling the three groups of branches. Finally, we consider the structural and performance constraints at the same time, which means we put $A_{S}, A_{P}$, and $b_{S}, b_{P}$ in one matrix. So, we obtain

$$
\begin{aligned}
& A=A_{S}+A_{P}=\left[\begin{array}{cccc}
l & A_{1} & & \\
l & & A_{2} & \\
l & & & A_{3} \\
A_{P} & &
\end{array}\right]_{12 \times 12}, \\
& b=b_{S}+b_{P}=\left[\begin{array}{l}
b_{1} \\
b_{2} \\
b_{3} \\
b_{P}
\end{array}\right]_{12 \times 1} .
\end{aligned}
$$

According to Udwadia-Kalaba approach, the closed-form control force can be expressed as

$$
\begin{aligned}
Q_{c} & =M^{1 / 2}\left(A M^{-1 / 2}\right)^{+}\left(b-A M^{-1} Q\right) \\
\tau= & M^{1 / 2}(q, t)\left(A(q, t) M^{-1 / 2}(q, t)\right)^{+} \\
& \cdot\left(b(q, \dot{q}, t)-A(q, t) M^{-1}(q, t) C(q, \dot{q}, t) \dot{q}\right) .
\end{aligned}
$$

Case 2. In Case 2, we let the movement of platform be more complex in order to verify the control approach. The movement in $x$ direction is uniform motion, in $y$ direction is trigonometric function, and in $z$ direction is parabola. Thus, the desired trajectory is given as follows.

$$
\begin{aligned}
& x_{p}=-\frac{2 \sqrt{3}}{3}-0.001 t \\
& y_{p}=2+R \sin (w t) \\
& z_{p}=-\frac{1}{250000} t^{2}+\frac{1}{250} t .
\end{aligned}
$$

Similarly, differentiating the constraint equation (37) with respect to $t$ twice, we can get

$$
\begin{aligned}
& \ddot{x}_{p}=0 \\
& \ddot{y}_{p}=-R w^{2} \sin (w t) \\
& \ddot{z}_{p}=-\frac{1}{125000} .
\end{aligned}
$$


TABLE 1: Parameters for simulation.

\begin{tabular}{lcc}
\hline Parameter & Name & Value \\
\hline$m_{e 1}, m_{e 2}, m_{e 3}$ & Mass of saddle & $10 \mathrm{~kg}$ \\
$m_{c 1}, m_{c 2}, m_{c 3}$ & Mass of branched chain. & $2 \mathrm{~kg}$ \\
$m_{P}$ & Mass of moving platform & $15 \mathrm{~kg}$ \\
$I_{1 x}, I_{1 y}, I_{2 x}, I_{2 y}, I_{3 x}, I_{3 y}$ & $m_{c 1} l^{2} / 3$ \\
$l$ & Moment of inertia around $x$ - and $y$-axis & $(4 \sqrt{6} / 3) \mathrm{m}$ \\
$R$ & Length of branched chain & $(\sqrt{3} / 5) \mathrm{m}$ \\
$S$ & Radius of constrained motion & $4 \mathrm{~m}$ \\
$\omega$ & Distance between two frame & $0.02 \pi$ \\
\hline
\end{tabular}

TABLE 2: Initial conditions of Case 1 for simulation.

\begin{tabular}{|c|c|c|c|c|c|c|c|}
\hline$h_{1}$ & $4 \sqrt{3} / 3$ & $h_{3}$ & $4 \sqrt{3} / 3$ & $\dot{h}_{1}$ & $-\sqrt{3} R \omega / 2$ & $\dot{h}_{3}$ & $\sqrt{3} R \omega / 2$ \\
\hline$\theta_{1}$ & $3 \pi / 4$ & $\theta_{3}$ & $3 \pi / 4$ & $\dot{\theta}_{1}$ & $-\sqrt{6} R \omega / 2 l$ & $\dot{\theta}_{3}$ & $\sqrt{6} R \omega / 2 l$ \\
\hline$\varphi_{1}$ & $2 \pi / 3$ & $\varphi_{3}$ & $4 \pi / 3$ & $\dot{\varphi}_{1}$ & $-\sqrt{2} R \omega / 2 l$ & $\dot{\varphi}_{3}$ & $-\sqrt{2} R \omega / 2 l$ \\
\hline$h_{2}$ & $4 \sqrt{3} / 3$ & $x_{p}$ & $-2 \sqrt{3} / 3$ & $\dot{h}_{2}$ & 0 & $\dot{x}_{p}$ & 0 \\
\hline$\theta_{2}$ & $3 \pi / 4$ & $y_{p}$ & 2 & $\dot{\theta}_{2}$ & 0 & $\dot{y}_{p}$ & $R \omega$ \\
\hline$\varphi_{2}$ & $2 \pi$ & $z_{p}$ & 0 & $\dot{\varphi}_{2}$ & $\sqrt{2} R \omega / l$ & $\dot{z}_{p}$ & 0.05 \\
\hline
\end{tabular}

Therefore, the matrices $A_{P}, b_{P}$ can be obtained. The control force can be obtained as well.

4.2. Simulation Results. In this section, the explicit analytic results given in Section 4 are verified by numerical simulations. The numerical integration through-out this paper is done in the MATLAB environment using a variable time step ode $15 \mathrm{i}$ integrator and the time of simulation is 1000 seconds. The values of the parameters are defined in Table 1.

We give the initial conditions of Case 1 including initial coordinate and velocity of the generalized coordinates in Table 2.

Figures 3 and 4 show the simulated trajectory of the parallel manipulator in different views. From the two figures, we can see the moving platform can move on the given trajectory by solving the Udwadia-Kalaba equation (36). In Figures 5, 6, and 7, we show the variation of the three parameters $h, \theta, \varphi$, respectively. Figure 8 proved the distance between the platform and three saddles is constant; in other words, the length of branched chains is unchangeable which means the method in this paper is right. Figures 9 and 10 show the error of the platform in $x$ and $y$ directions, respectively. The errors are really small which means the platform can move along the desired trajectory. Figure 11 shows the forces generated by motors which verified the new approach validity.

In Case 2, we give the initial conditions in Table 3.

Figures 12 and 13 show the simulated trajectory in Case 2. From the Figures 13(a), 13(b), and 13(c), we can see the trajectory in $x, y$, and $z$ directions clearly. Figures 14, 15, and 16 show the variation of the three parameters $h, \theta, \varphi$, respectively. Figures 17,18 , and 19 show the error of the platform in $x, y$, and $z$ directions, respectively. The errors are

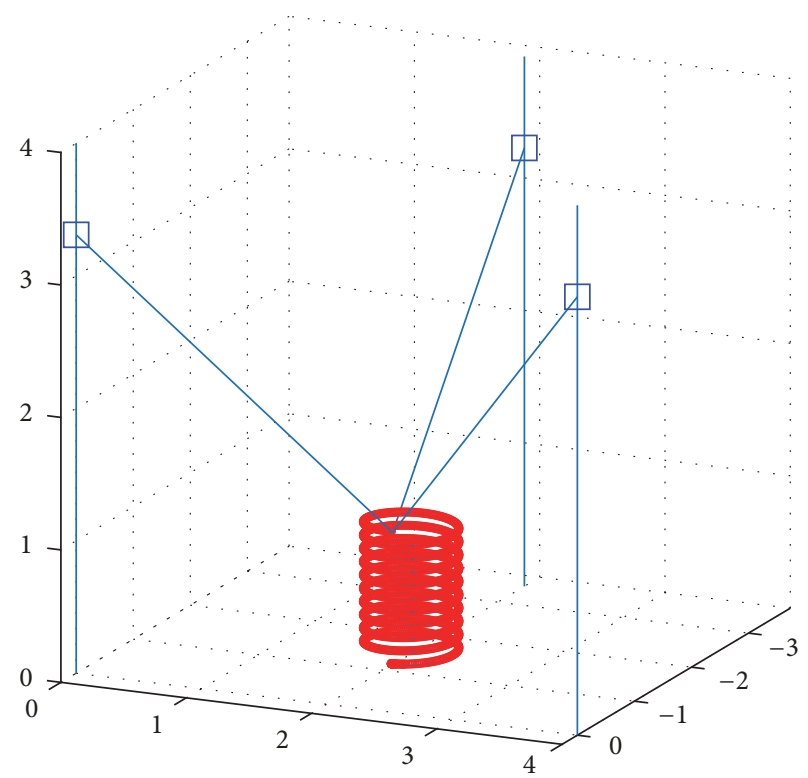

FIGURE 3: The simulated trajectory of the parallel manipulator in Case 1.

all very small which are, respectively, in the order of $10^{-11}$, $10^{-5}$, and $10^{-10}$. Figure 20 shows the forces generated by motors in Case 2.

\section{Conclusion}

In this paper, Udwadia-Kalaba approach is applied for trajectory tracking control of the 3-HSS parallel manipulator for the first time. First, we establish the nonlinear dynamic 
TABLE 3: Initial conditions of Case 2 for simulation.

\begin{tabular}{cccccccc}
\hline$h_{1}$ & $4 \sqrt{3} / 3$ & $h_{3}$ & $4 \sqrt{3} / 3$ & $\dot{h}_{1}$ & $-\sqrt{3} R \omega / 2$ & $\dot{h}_{3}$ & $\sqrt{3} R \omega / 2$ \\
$\theta_{1}$ & $3 \pi / 4$ & $\theta_{3}$ & $3 \pi / 4$ & $\dot{\theta}_{1}$ & $-\sqrt{6} R \omega / 2 l$ & $\dot{\theta}_{3}$ & $\sqrt{6} R \omega / 2 l$ \\
$\varphi_{1}$ & $2 \pi / 3$ & $\varphi_{3}$ & $4 \pi / 3$ & $\dot{\varphi}_{1}$ & $-\sqrt{2} R \omega / 2 l$ & $\dot{\varphi}_{3}$ & $-\sqrt{2} R \omega / 2 l$ \\
$h_{2}$ & $4 \sqrt{3} / 3$ & $x_{p}$ & $-2 \sqrt{3} / 3$ & $\dot{h}_{2}$ & 0 & $\dot{x}_{p}$ & -0.001 \\
$\theta_{2}$ & $3 \pi / 4$ & $y_{p}$ & 2 & $\dot{\theta}_{2}$ & 0 & $\dot{y}_{p}$ & $R \omega$ \\
$\varphi_{2}$ & $2 \pi$ & $z_{p}$ & 0 & $\dot{\varphi}_{2}$ & $\sqrt{2} R \omega / l$ & $\dot{z}_{p}$ & $1 / 250$ \\
\hline
\end{tabular}

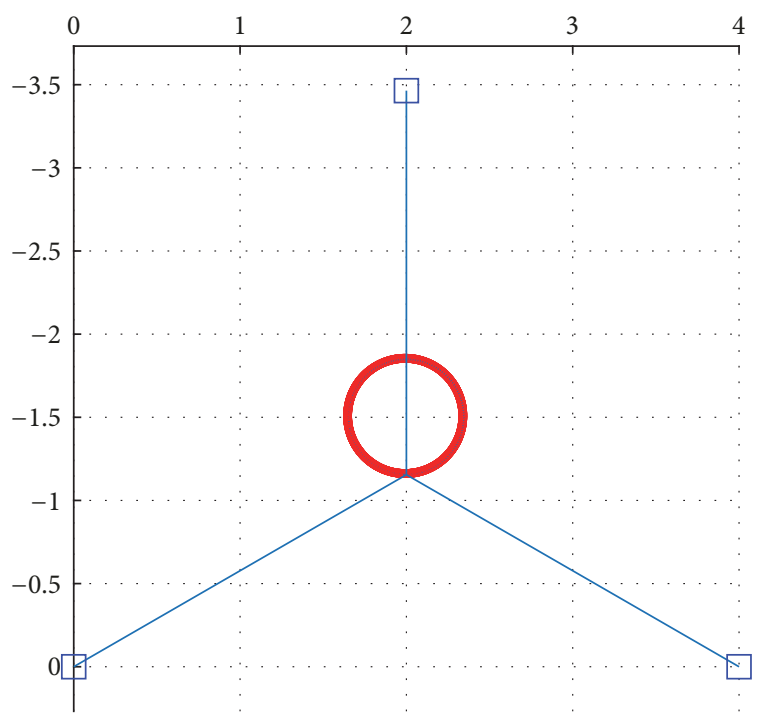

Figure 4: The simulated trajectory of the parallel manipulator in different view in Case 1.

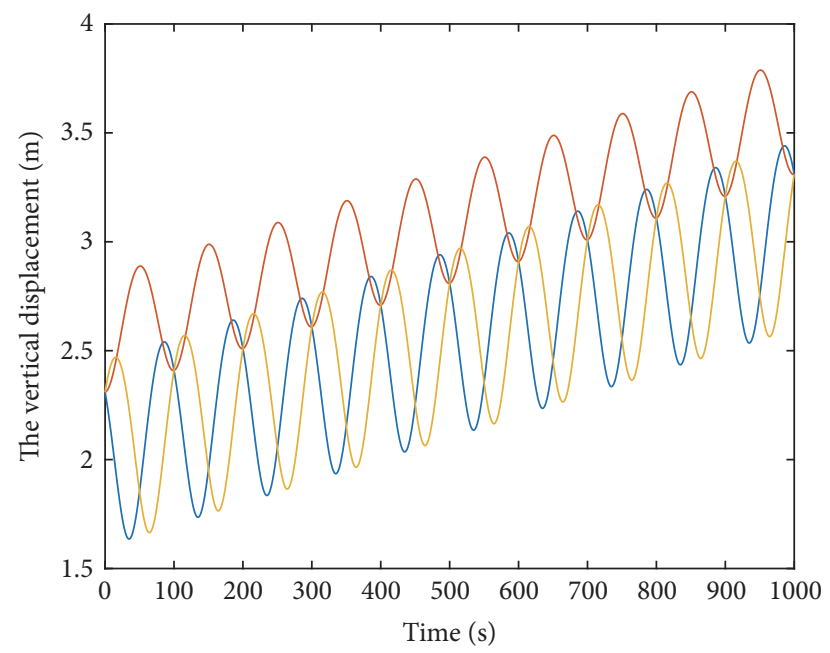

Figure 5: The variation of parameter $h$ in simulation in Case 1.

model by dividing the system into four "unconstrained" subsystems which simplifies the modeling process. Next, the system structure and desired trajectory are regarded as the

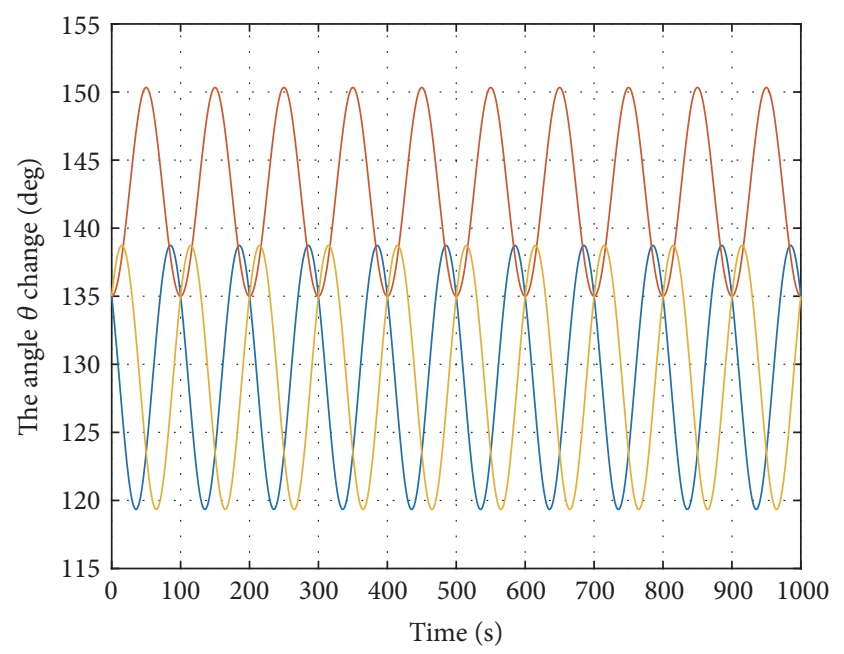

FIgURE 6: The variation of parameter $\theta$ in simulation in Case 1.

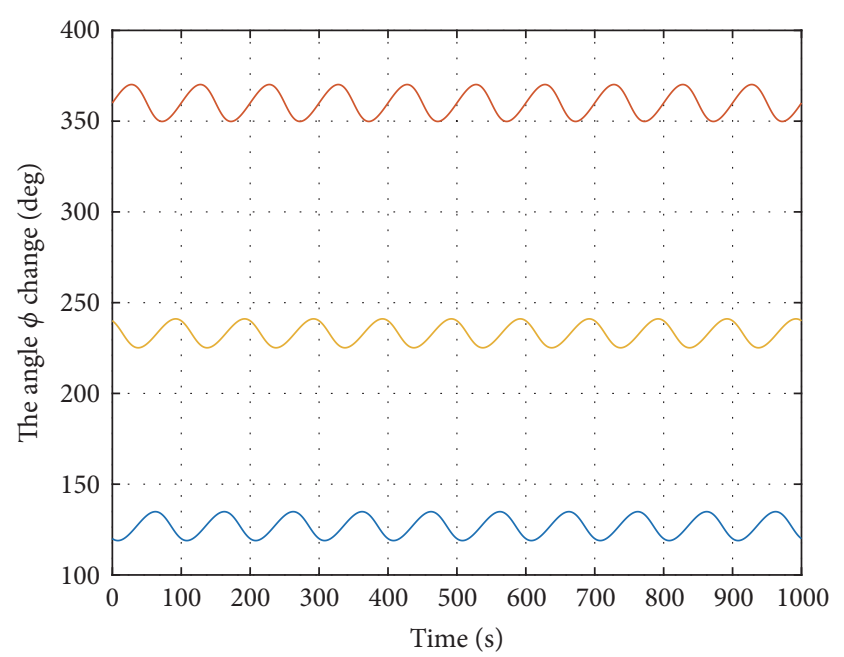

FIgURE 7: The variation of parameter $\varphi$ in simulation in Case 1.

structure constraints and performance constraints, respectively. Finally, this approach provides an explicit, closedform analytical expression for the control force by solving Udwadia-Kalaba equation. The simulation results of two cases show that the servoconstraint forces based on UdwadiaKalaba equation make the platform's movement meets the designed trajectory precisely. 


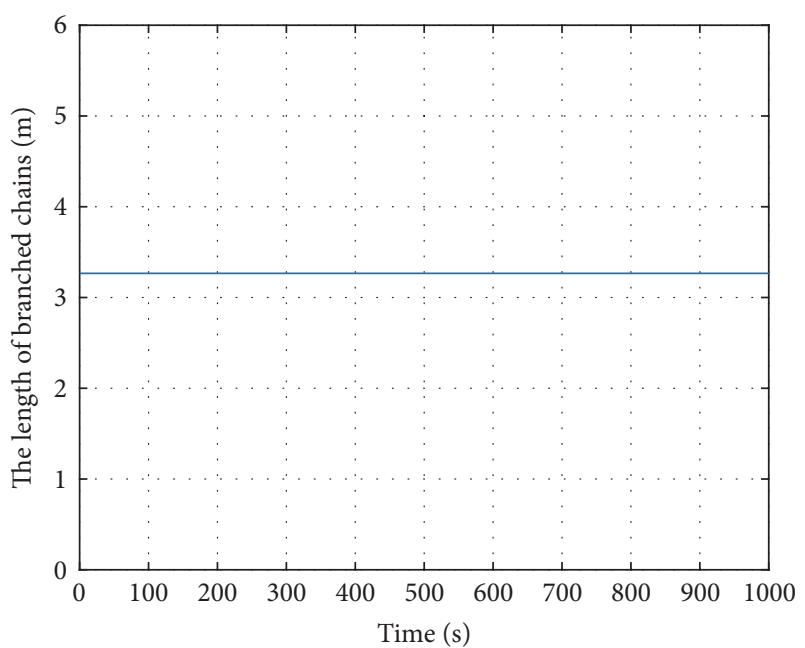

FIGURE 8: The variation of branched chains' length in simulation in Case 1.

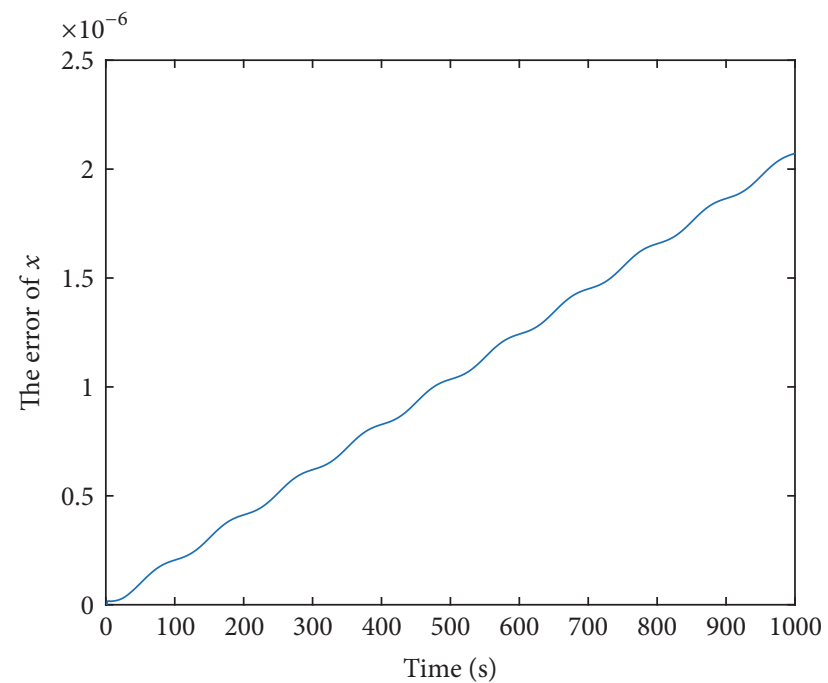

FIGURE 9: The error of platform in the $x$ direction in Case 1.

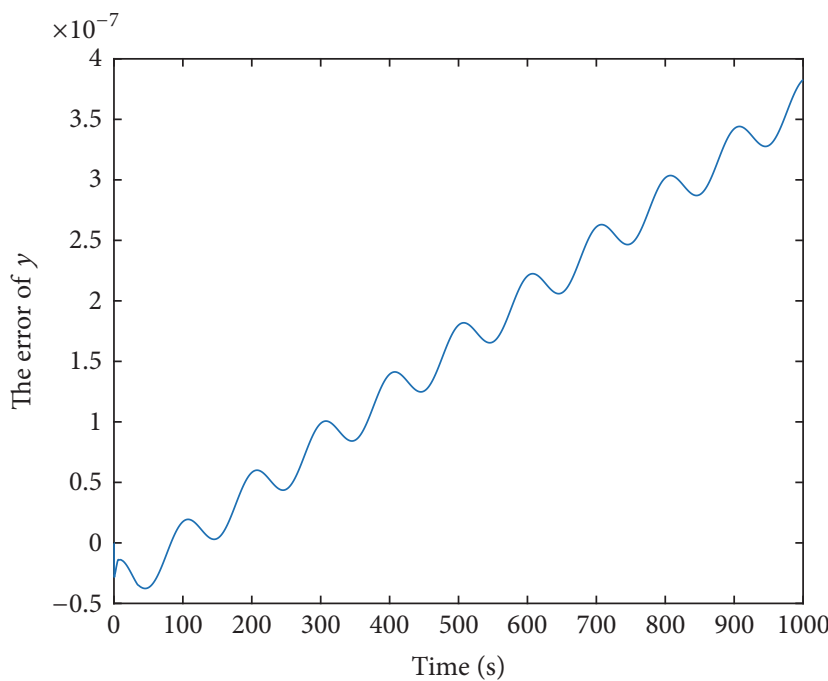

Figure 10: The error of platform in the $y$ direction in Case 1.

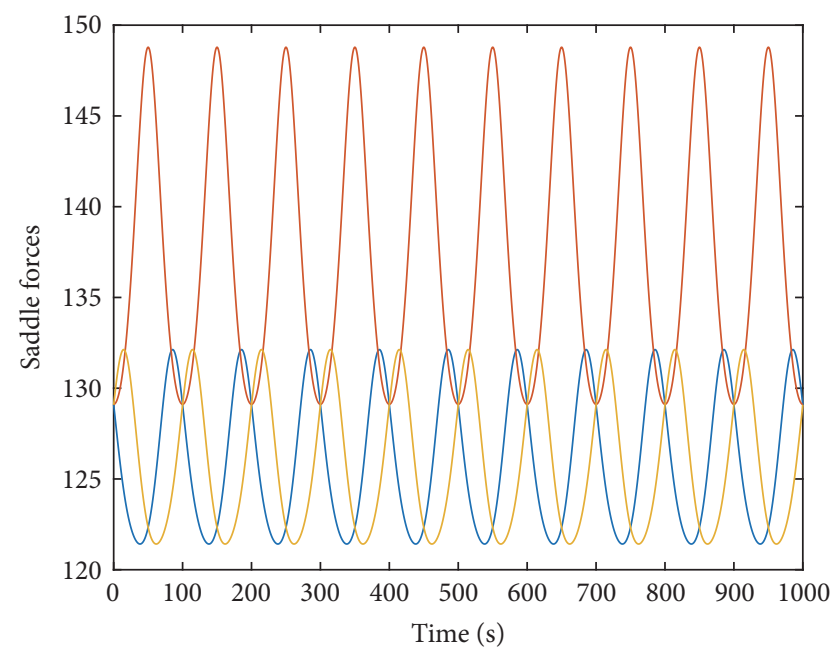

FIGURE 11: The simulated lift forces generated by motors as functions of time in Case 1.

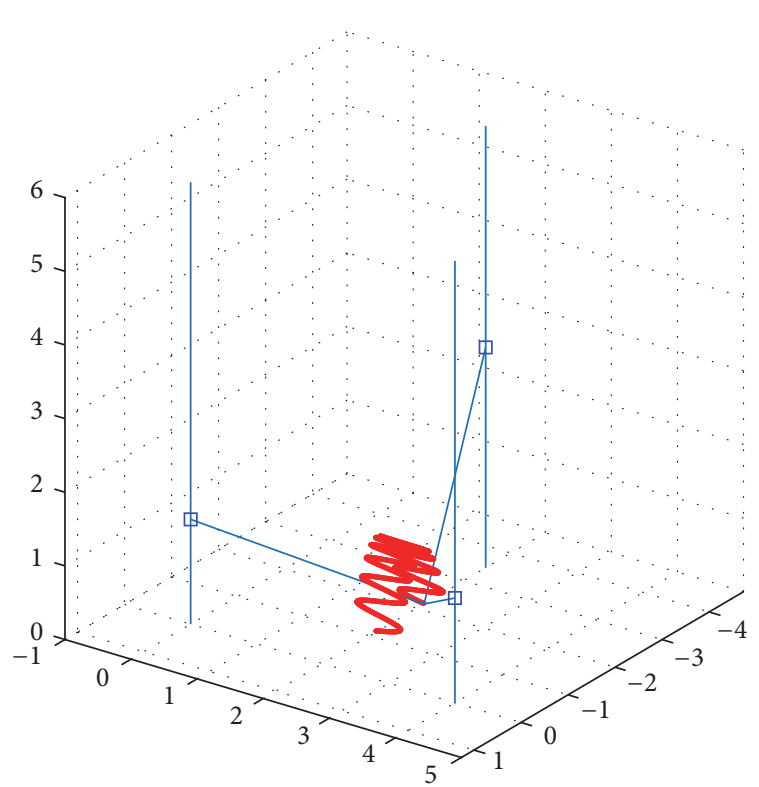

FIGURE 12: The simulated trajectory of the parallel manipulator in Case 2.

In summary, there are two main advantages of this approach in this paper. First, this paper provides an easy dynamic modeling process for 3-HSS parallel manipulator. The constraints in the system which can be holonomic as well as nonholonomic are divided into structure constraints and performance constraints. What we need do is to get the second-order-form constraints. It is more convenient compared with the common approach. Second, the control performance is excellent due to the exact equation of motion by this approach. However, in another approach, there are approximations of the equation of motion or modification of the controller such as Lagrange multiplier which makes the equation complicated and the tracking performance not optimistic. 


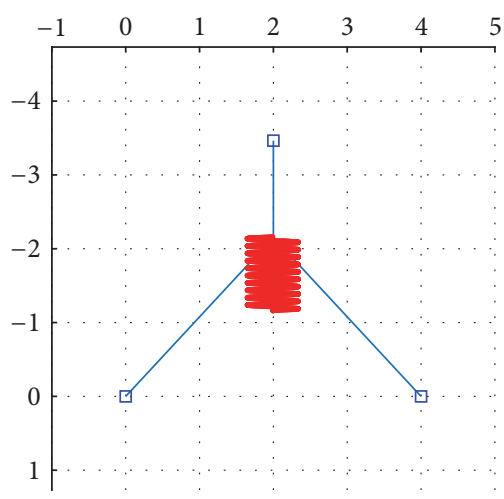

(a) The projection on $x-y$ plane

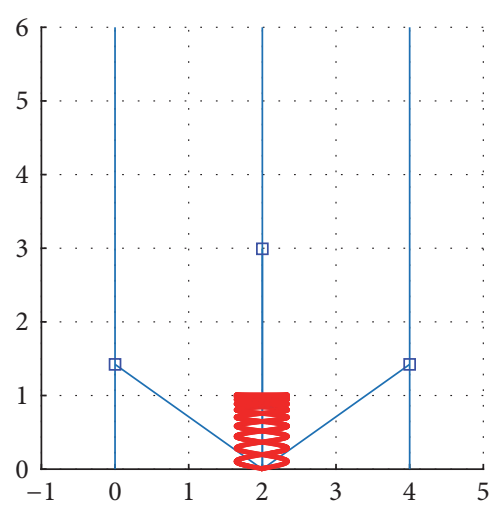

(b) The projection on $y-z$ plane

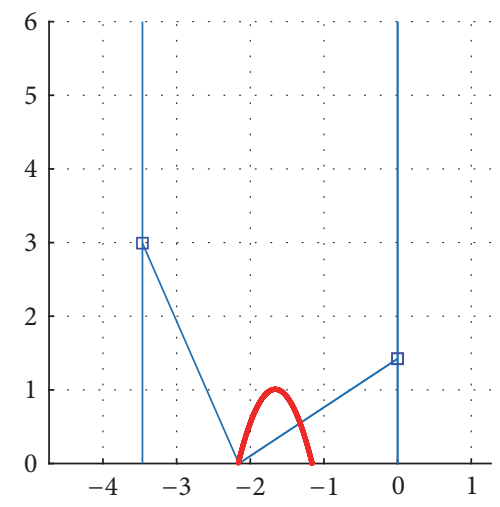

(c) The projection on $x-z$ plane

FIGURE 13: The simulated trajectory of the parallel manipulator in different views in Case 2.

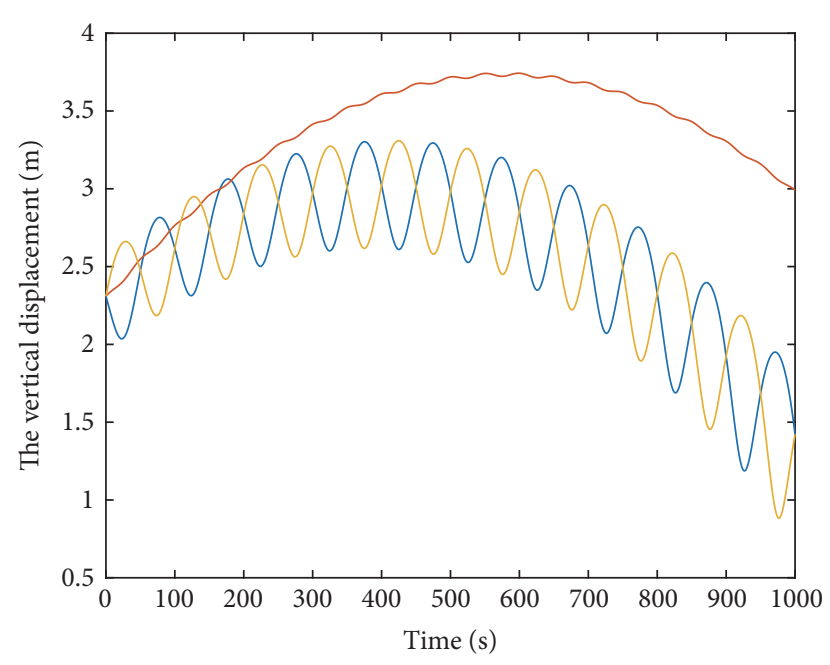

FIGURE 14: The variation of parameter $h$ in simulation in Case 2.

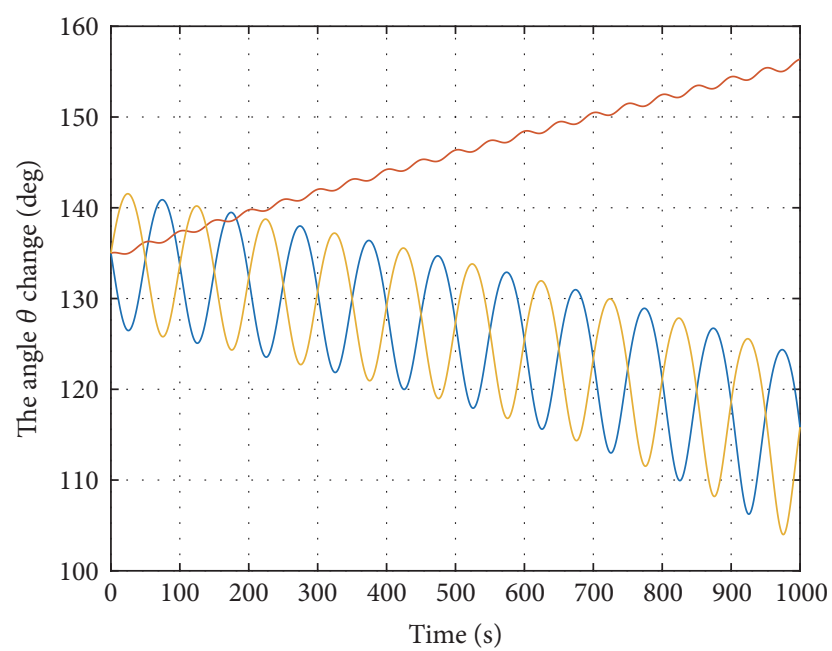

FIGURE 15: The variation of parameter $\theta$ in simulation in Case 2.

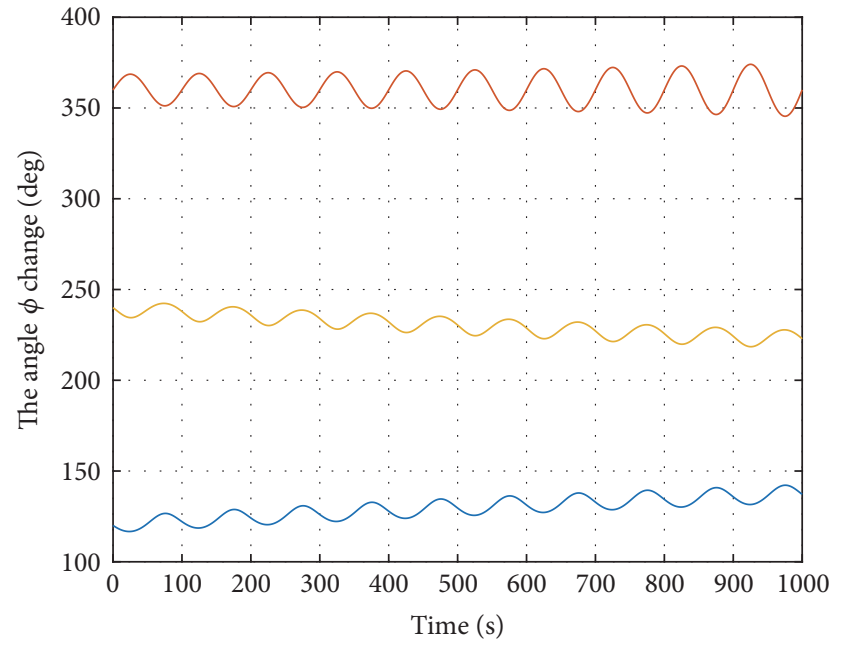

FIGURE 16: The variation of parameter $\varphi$ in simulation in Case 2.

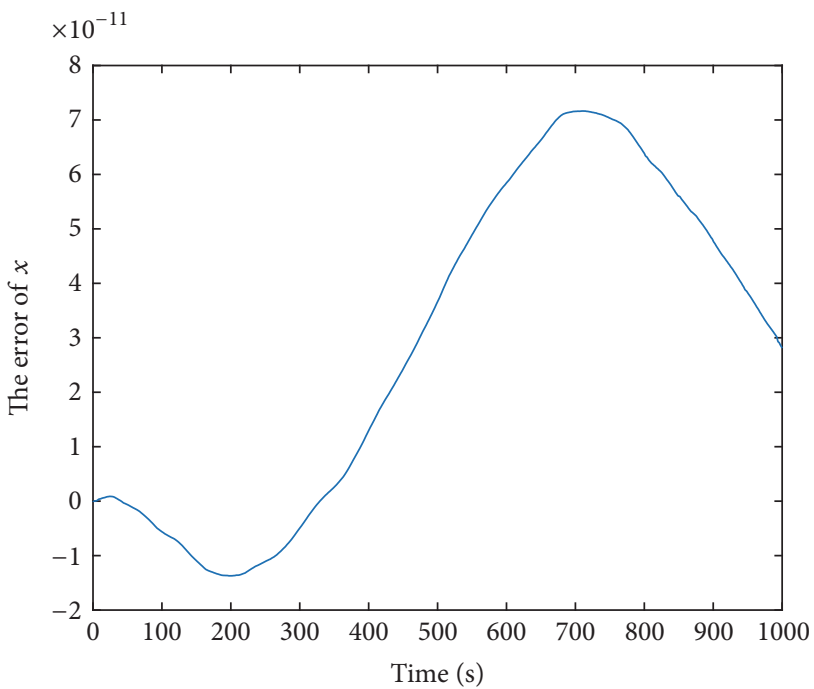

FIGURE 17: The error of platform in the $x$ direction in Case 2. 


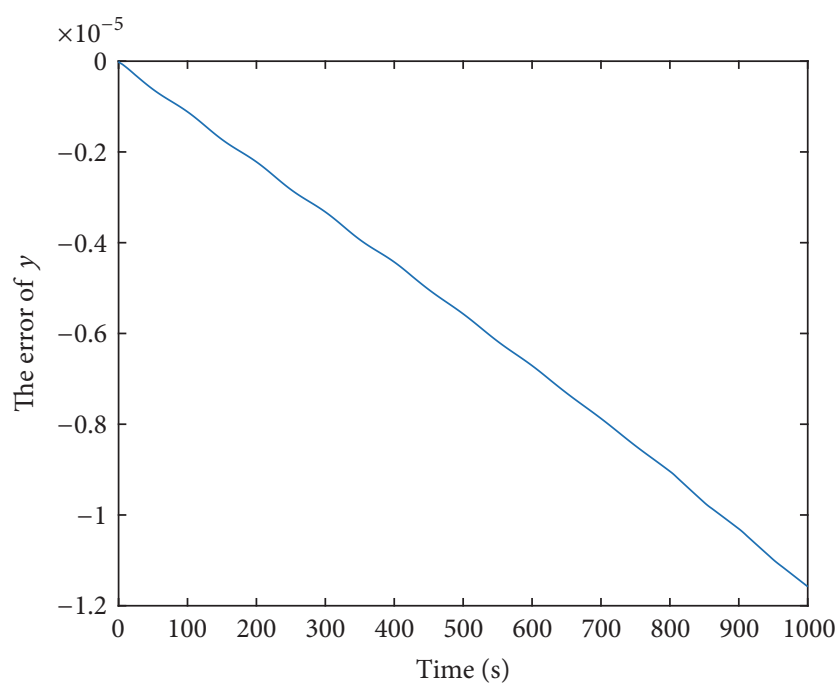

FIGURE 18: The error of platform in the $y$ direction in Case 2.

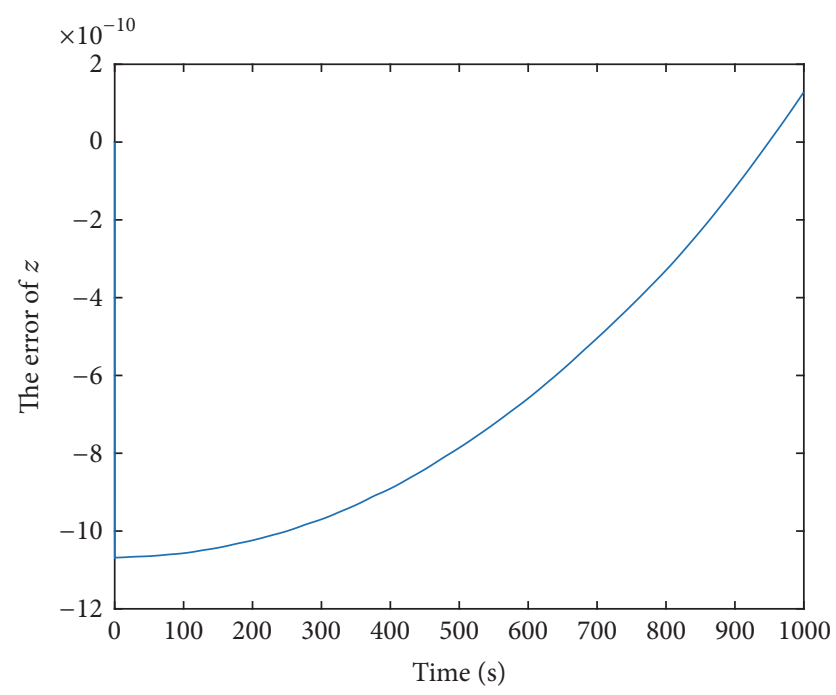

FIGURE 19: The error of platform in the $z$ direction in Case 2.

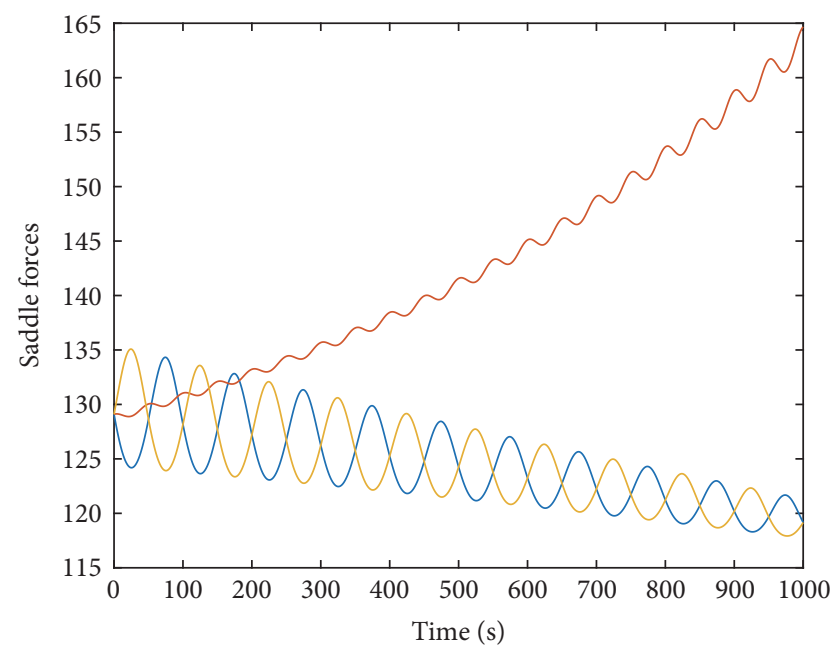

Figure 20: The simulated lift forces generated by motors as functions of time in Case 2.

\section{Conflicts of Interest}

The authors declare that they have no conflicts of interest.

\section{Acknowledgments}

The theoretical foundation of this paper is consistent with (1) National Natural Science Foundation of China (51505116); (2) the Fundamental Research Funds for the Central Universities (JZ2016HGTB0716); (3) Natural and Science Foundation of Anhui Province (1508085SME221); (4) China Postdoctoral Science Foundation (2016M590563). In addition, the project is supported by these foundations.

\section{References}

[1] J. P. Merlet, Parallel Robots, Kluwer Academic Publishers, Dordrecht, The Netherlands, 2006.

[2] J. Wu, J. Wang, T. Li, and L. Wang, "Performance analysis and application of a redundantly actuated parallel manipulator for milling," Journal of Intelligent \& Robotic Systems, vol. 50, no. 2, pp. 163-180, 2007.

[3] J. Kim, F. C. Park, S. J. Ryu et al., "Design and analysis of a redundantly actuated parallel mechanism for rapid machining," IEEE Transactions on Robotics and Automation, vol. 17, no. 4, pp. 423-434, 2001.

[4] Y. Li and Q. Xu, "Design and development of a medical parallel robot for cardiopulmonary resuscitation," IEEE/ASME Transactions on Mechatronics, vol. 12, no. 3, pp. 265-273, 2007.

[5] Y. Su, D. Sun, L. Ren, and J. K. Mills, "Integration of saturated PI synchronous control and PD feedback for control of parallel manipulators," IEEE Transactions on Robotics, vol. 22, no. 1, pp. 202-207, 2006

[6] N.-I. Kim, C.-W. Lee, and P.-H. Chang, "Sliding mode control with perturbation estimation: Application to motion control of parallel manipulator," Control Engineering Practice, vol. 6, no. 11, pp. 1321-1330, 1998.

[7] W. Shang and S. Cong, "Nonlinear adaptive task space control for a 2-DOF redundantly actuated parallel manipulator," Nonlinear Dynamics, vol. 59, no. 1-2, pp. 61-72, 2010.

[8] V. Vinoth, Y. Singh, and M. Santhakumar, "Indirect disturbance compensation control of a planar parallel (2-PRP and 1-PPR) robotic manipulator," Robotics and Computer-Integrated Manufacturing, vol. 30, no. 5, pp. 556-564, 2014.

[9] K. Fu and J. K. Mills, "Robust control design for a planar parallel robot," International Journal of Robotics \& Automation, vol. 22, no. 2, pp. 139-147, 2007.

[10] W. W. Shang and S. Cong, "Robust nonlinear control of a planar 2-DOF parallel manipulator with redundant actuation," Robotics and Computer-Integrated Manufacturing, vol. 30, no. 6, pp. 597-604, 2014.

[11] M. A. Khosravi and H. D. Taghirad, "Robust PID control of fully-constrained cable driven parallel robots," Mechatronics, vol. 24, no. 2, pp. 87-97, 2014.

[12] M. Zeinali and L. Notash, "Adaptive sliding mode control with uncertainty estimator for robot manipulators," Mechanism and Machine Theory, vol. 45, no. 1, pp. 80-90, 2010.

[13] X. Zhu, G. Tao, B. Yao, and J. Cao, "Adaptive robust posture control of parallel manipulator driven by pneumatic muscles with redundancy," IEEE/ASME Transactions on Mechatronics, vol. 13, no. 4, pp. 441-450, 2008. 
[14] A. Noshadi, M. Mailah, and A. Zolfagharian, "Intelligent active force control of a 3-RRR parallel manipulator incorporating fuzzy resolved acceleration control," Applied Mathematical Modelling, vol. 36, no. 6, pp. 2370-2383, 2012.

[15] H. Sadjadian, H. D. Taghirad, and A. Fatehi, "Neural networks approaches for computing the forward kinematics of a redundant parallel manipulator," International Journal of Computational Intelligence, vol. 2, no. 1, pp. 40-47, 2005.

[16] D. Zhu and Y. Fang, "Adaptive control of parallel manipulators via fuzzy-neural network algorithm," Control Theory and Technology, vol. 5, no. 3, pp. 295-300, 2007.

[17] F. E. Udwadia and R. E. Kalaba, "A new perspective on constrained motion," Proceedings: Mathematical and Physical Sciences, vol. 439, no. 1906, pp. 407-410, 1992.

[18] F. E. Udwadia and R. E. Kalaba, Analytical dynamics: A new approach, Cambridge University Press, Cambridge, UK, 1996.

[19] F. E. Udwadia and R. E. Kalaba, "Explicit equations of motion for mechanical systems with nonideal constraints," Journal of Applied Mechanics, vol. 68, no. 3, pp. 462-467, 2001.

[20] F. E. Udwadia and R. E. Kalaba, "What is the general form of the explicit equations of motion for constrained mechanical systems?" Journal of Applied Mechanics, vol. 69, no. 3, pp. 335339, 2002. 


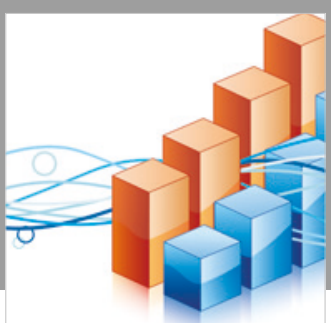

Advances in

Operations Research

\section{-n-m}
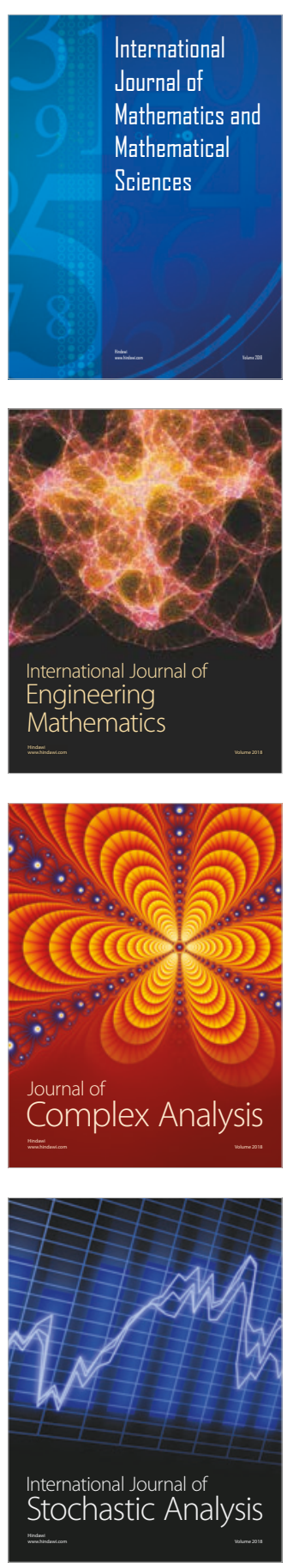
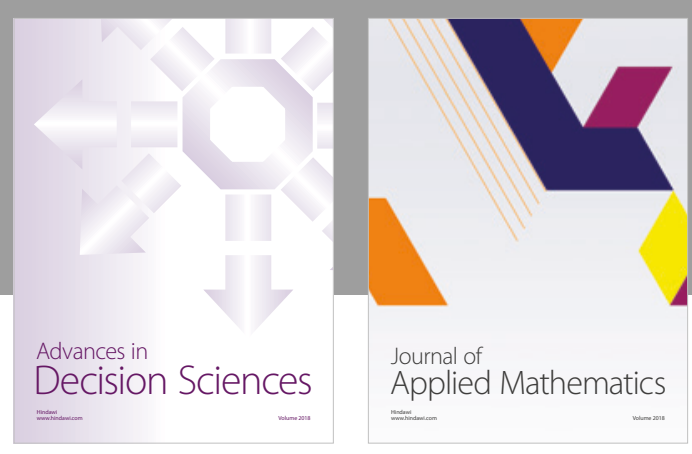

Journal of

Applied Mathematics
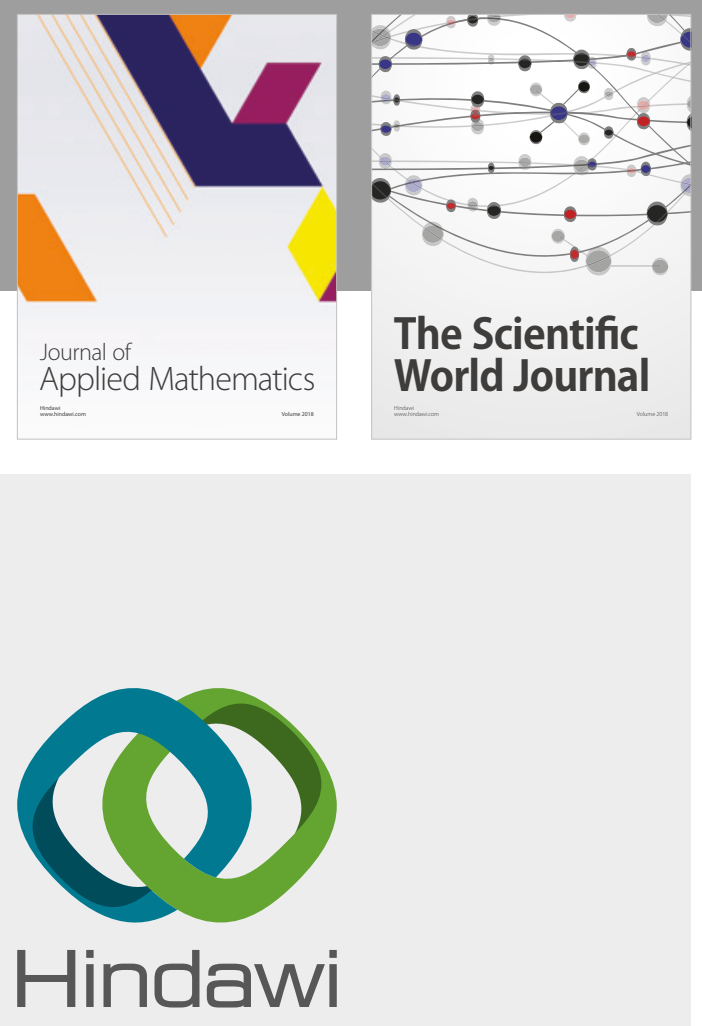

Submit your manuscripts at

www.hindawi.com

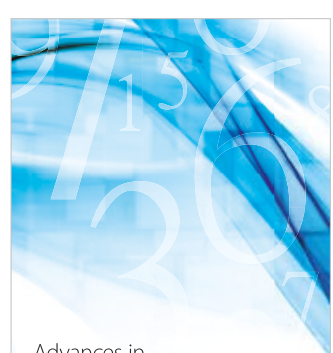

Advances in
Numerical Analysis
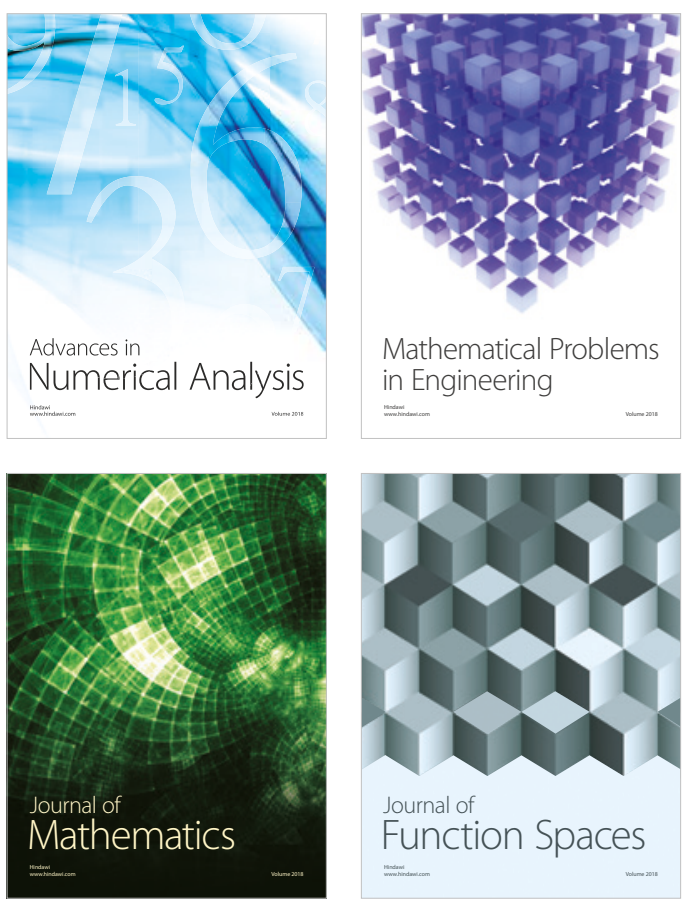

Mathematical Problems in Engineering

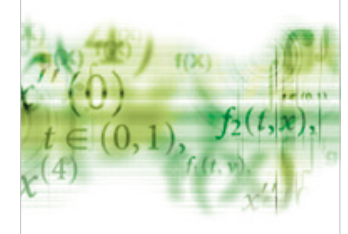

International Journal of

Differential Equations

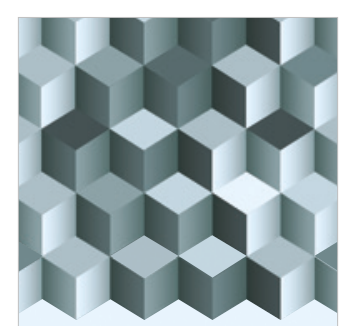

Journal of

Function Spaces

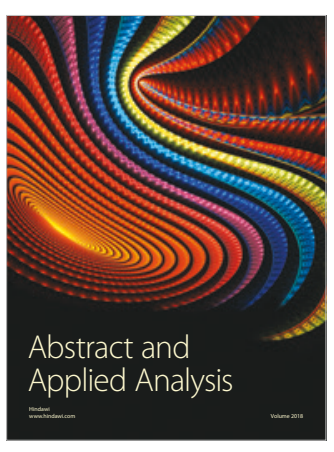

The Scientific

World Journal

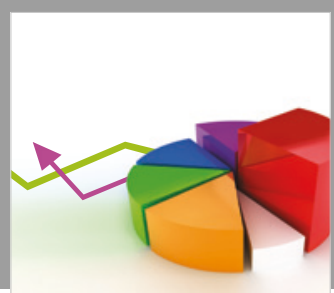

Journal of

Probability and Statistics
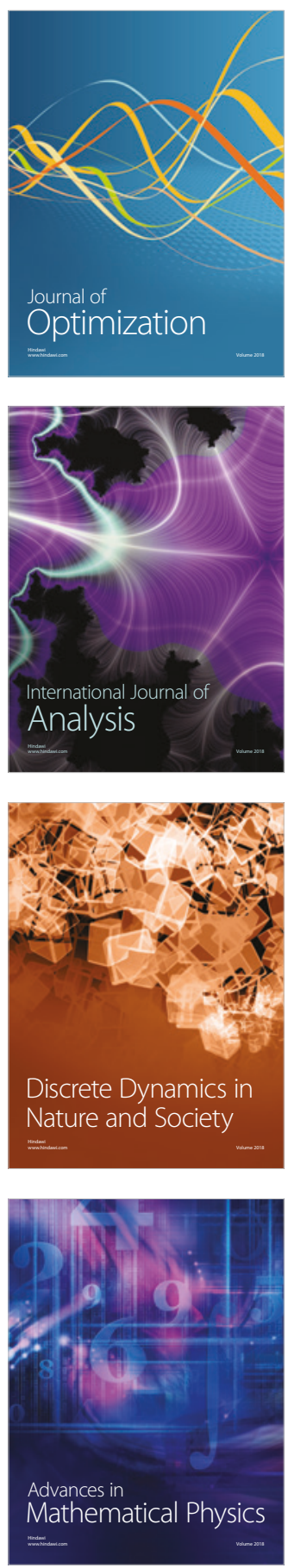